\title{
Genetic and epigenetic factors are associated with expression of respiratory chain component NDUFB6 in human skeletal muscle
}

\author{
Charlotte Ling, ${ }^{1}$ Pernille Poulsen, ${ }^{2}$ Stina Simonsson, ${ }^{3}$ Tina Rönn, ${ }^{1}$ Johan Holmkvist, ${ }^{1}$ Peter Almgren, ${ }^{1}$
} Per Hagert, ${ }^{1}$ Emma Nilsson, ${ }^{2}$ Amanda G. Mabey, ${ }^{1}$ Peter Nilsson, ${ }^{1}$ Allan Vaag, ${ }^{1,2}$ and Leif Groop ${ }^{1}$

1Department of Clinical Sciences, Lund University, University Hospital MAS, Malmö, Sweden. ${ }^{2}$ Steno Diabetes Center, Gentofte, Denmark. 3Institute of Biomedicine, Sahlgrenska Academy, Göteborg University, Göteborg, Sweden.

\begin{abstract}
Insulin resistance and type 2 diabetes are associated with decreased expression of genes that regulate oxidative phosphorylation in skeletal muscle. To determine whether this defect might be inherited or acquired, we investigated the association of genetic, epigenetic, and nongenetic factors with expression of NDUFB6, a component of the respiratory chain that is decreased in muscle from diabetic patients. Expression of NDUFB6 was influenced by age, with lower gene expression in muscle of elderly subjects. Heritability of NDUFB6 expression in muscle was estimated to be approximately $60 \%$ in twins. A polymorphism in the NDUFB6 promoter region that creates a possible DNA methylation site $(\mathrm{rs629566,A/G)}$ was associated with a decline in muscle NDUFB6 expression with age. Although young subjects with the rs $629566 \mathrm{G} / \mathrm{G}$ genotype exhibited higher muscle NDUFB6 expression, this genotype was associated with reduced expression in elderly subjects. This was subsequently explained by the finding of increased DNA methylation in the promoter of elderly, but not young, subjects carrying the rs629566 G/G genotype. Furthermore, the degree of DNA methylation correlated negatively with muscle NDUFB6 expression, which in turn was associated with insulin sensitivity. Our results demonstrate that genetic, epigenetic, and nongenetic factors associate with NDUFB6 expression in human muscle and suggest that genetic and epigenetic factors may interact to increase age-dependent susceptibility to insulin resistance.
\end{abstract}

\section{Introduction}

Although reduced physical activity, obesity, and aging increase susceptibility to type 2 diabetes mellitus (T2DM), not all individuals exposed to these risk factors develop the disease. A likely reason is that genetic variation modifies susceptibility to T2DM. Furthermore, the interaction between genetic and nongenetic factors may be even more complex and involve epigenetic factors such as DNA methylation. Insulin-resistant offspring of patients with T2DM and elderly subjects are characterized by impaired mitochondrial function in skeletal muscle $(1,2)$. Furthermore, nuclear-encoded genes regulating oxidative phosphorylation (OXPHOS), and their transcriptional regulators, PPAR $\gamma$ coactivator $1 \alpha(P G C-1 \alpha)$ and $P G C-1 \beta$, show reduced expression in skeletal muscle of patients with $\operatorname{T} 2 \operatorname{DM}(3,4)$. Nevertheless, it remains unknown whether this is an inherited or acquired defect. We previously showed that an age-dependent decrease in muscle $P G C-1 \alpha$ and $P G C$ - $1 \beta$ expression is partially under genetic control and is influenced by the $P G C-1 \alpha$ Gly482Ser polymorphism (5). This common variant in the $P G C-1 \alpha$ gene has also been associated with increased risk of T2DM (6-8). We hypothesized that the effect of age on mitochondrial function can be determined by both genetic and epigenetic factors. To address this we selected the NDUFB6 gene from the first respiratory complex for further studies, because NDUFB6 is among the set of OXPHOS genes showing significant reduction in muscle from patients with T2DM compared with healthy control subjects and

Nonstandard abbreviations used: GEE, generalized estimating equation; LD, linkage disequilibrium; OXPHOS, oxidative phosphorylation; PGC-1, PPAR $\gamma$ coactivator 1; T2DM, type 2 diabetes mellitus; $\mathrm{VO}_{2} \max$, total body aerobic capacity. Conflict of interest: The authors have declared that no conflict of interest exists. Citation for this article: J. Clin. Invest. 117:3427-3435 (2007). doi:10.1172/JCI30938. because the first complex has a key role in regulating OXPHOS $(3,9)$. We specifically examined whether polymorphisms in the promoter of the gene were associated with an age-dependent impact on gene expression and/or risk of T2DM and whether this was modified by epigenetic factors like DNA methylation. To accomplish this we studied muscle biopsies obtained from young and elderly twins and we used a case-control approach to study the impact of genetic variation in NDUFB6 on risk of T2DM.

Here we demonstrated that combinations of genetic and epigenetic factors were associated with the reduction in NDUFB6 expression in skeletal muscle from elderly compared with young twins. A polymorphism (rs629566, A/G) in the promoter region of NDUFB 6 that creates a possible methylation site was associated with the age-related decline in muscle NDUFB6 expression. We demonstrated that elderly subjects carrying the rs629566 G/G genotype had reduced NDUFB6 expression together with increased DNA methylation of the NDUFB6 promoter; the degree of DNA methylation correlated negatively with the expression level of NDUFB6. Furthermore, the level of NDUFB6 in human skeletal muscle was associated with insulin sensitivity. Our study provides a scenario by which genetic and epigenetic factors may interact to increase age-dependent susceptibility to insulin resistance.

\section{Results}

Expression of NDUFB6 in skeletal muscle decreases with age. As expected, elderly twins had higher BMI and lower total body aerobic capacity $\left(\mathrm{VO}_{2} \max \right)$ than younger twins (Table 1) (5). The relative mRNA levels of NDUFB6 were significantly reduced in muscle biopsies from elderly compared with young twins, both in the basal $(0.28 \pm 0.01$ versus $0.36 \pm 0.01 ; P<0.0005)$ and insulin-stimulated state $(0.33 \pm 0.01$ versus $0.39 \pm 0.01 ; P<0.007$; Figure $1 \mathrm{~A})$. 
Table 1

Clinical characteristics of participating subjects

\begin{tabular}{|c|c|c|c|c|c|c|}
\hline & \multicolumn{2}{|c|}{ Twins } & \multicolumn{2}{|c|}{ Screening set (Botnia) } & \multicolumn{2}{|c|}{ Replication set (Malmö) } \\
\hline & Young & Elderly & Cases & Controls & Cases & Controls \\
\hline$n$ (male/female) & $110(60 / 50)$ & $86(38 / 48)$ & $751(399 / 352)$ & $715(345 / 370)$ & $2,830(1,667 / 1,163)$ & $3,740(1,417 / 2,323)$ \\
\hline$n(\mathrm{MZ} / \mathrm{DZ})$ & $110(66 / 44)$ & $86(42 / 44)$ & & & & \\
\hline Age (yr) & $28.0 \pm 1.9$ & $62.4 \pm 2.0^{\mathrm{A}}$ & $60.3 \pm 9.9$ & $53.7 \pm 11.4^{\mathrm{A}}$ & $57.9 \pm 11.5$ & $57.4 \pm 6.0$ \\
\hline BMI $\left(\mathrm{kg} / \mathrm{m}^{2}\right)$ & $24.1 \pm 3.1$ & $26.1 \pm 4.4^{\mathrm{A}}$ & $28.9 \pm 4.8$ & $25.8 \pm 3.7^{\mathrm{A}}$ & $29.6 \pm 5.5$ & $25.1 \pm 3.6^{A}$ \\
\hline WHR & $0.84 \pm 0.08$ & $0.89 \pm 0.1^{A}$ & $0.94 \pm 0.09$ & $0.87 \pm 0.09 \mathrm{~A}$ & - & $0.84 \pm 0.09$ \\
\hline LBM (kg) & $55.7 \pm 11.9$ & $50.2 \pm 12.5^{\mathrm{A}}$ & $57.1 \pm 11.0$ & $54.0 \pm 10.4 \mathrm{~A}$ & - & - \\
\hline Body fat $(\%)$ & $22.0 \pm 7.0$ & $27.9 \pm 9.4 \mathrm{~A}$ & $30.2 \pm 8.1$ & $27.8 \pm 7.7^{A}$ & - & - \\
\hline $\mathrm{VO}_{2} \max (\mathrm{ml} / \mathrm{kg} / \mathrm{min})$ & $39.6 \pm 7.8$ & $26.3 \pm 6.9^{A}$ & - & - & - & - \\
\hline \multicolumn{3}{|c|}{ Fasting plasma glucose (mM) } & $9.1 \pm 3.2$ & $5.3 \pm 0.5^{A}$ & $11.9 \pm 4.3$ & $5.4 \pm 0.4^{A}$ \\
\hline \multicolumn{3}{|c|}{ Plasma glucose (2 h; mM) } & $13.8 \pm 5.8$ & $5.2 \pm 1.2^{\mathrm{A}}$ & - & - \\
\hline \multicolumn{3}{|c|}{ Fasting serum insulin (mU/l) } & $15.2 \pm 11.3$ & $7.0 \pm 3.8^{A}$ & - & $6.5 \pm 4.1$ \\
\hline \multicolumn{3}{|c|}{ Serum insulin (2 h; mU/l) } & $66.4 \pm 55.6$ & $33.4 \pm 25.9 \mathrm{~A}$ & - & - \\
\hline
\end{tabular}

DZ, dizygotic; LBM, lean body mass; MZ, monozygotic; WHR, waist/hip ratio. Data are mean \pm SD. In order to adjust for the lack of independence between monozygotic and dizygotic twins, all comparisons of mean differences between age groups were performed using GEE methodology. ${ }^{A} P<0.05$.

Furthermore, insulin caused a modest but significant increase in the mRNA level of NDUFB6 in both young and elderly twins $(P<0.0001$ and $P<0.01$, respectively; Figure $1 \mathrm{~A})$. NDUFB6 mRNA levels correlated with those of protein in muscle biopsies from 8 young and 8 elderly twins, selected randomly, before $(r=0.71$; $P<0.005)$ and after $(r=0.61 ; P<0.02)$ insulin stimulation (Figure 1, $\mathrm{D}$ and $\mathrm{E})$. In agreement with the mRNA findings, the relative protein levels of NDUFB 6 were significantly lower in elderly than in young twins, both in the basal $(0.54 \pm 0.044$ versus $0.66 \pm 0.028 ; P<0.05)$ and insulin-stimulated states $(0.52 \pm 0.041$ versus $0.66 \pm 0.029$; $P<0.05$; Figure $1, \mathrm{~B}$ and $\mathrm{C}$ ).

Expression of NDUFB6 in skeletal muscle is under genetic control. We estimated the degree of genetic and nongenetic influence on muscle NDUFB6 expression by biometric modeling (Table 2). In both young and elderly twins, NDUFB6 expression was under genetic influence with a heritability of $52 \%-65 \%$ (Table 2 ). However, the biometric model showed that both genetic and nongenetic factors influenced NDUFB6 expression.

$P G C-1 \alpha$ is associated with NDUFB6 expression in buman muscle. Because $P G C-1 \alpha$ is a key regulator of OXPHOS genes, we investigated whether the expression of PGC-1 $\alpha$ was related to NDUFB6 expression in muscle of 87 young and 68 elderly twins using a multivariate regression analysis including age, sex, and BMI as covariates. There was a significant positive association between insulin-stimulated PGC-1 $\alpha$ mRNA levels and NDUFB6 expression in

\section{Figure 1}

Effects of age and insulin on human skeletal muscle NDUFB6 levels. (A) Skeletal muscle biopsies were taken from young $(n=91)$ and elderly $(n=70)$ twins, before and after a hyperinsulinemic clamp. RNA was analyzed for NDUFB6 mRNA expression together with the internal standard cyclophilin A. The NDUFB6/cyclophilin A ratio was calculated for each sample. In order to adjust for the lack of independence between monozygotic and dizygotic twins, mean differences between groups were compared using GEE methodology. (B and C) The effect of age on NDUFB6 protein levels before (B) and after (C) insulin stimulation was analyzed by Western blot in 8 young and 8 elderly twins. (D and E) Correlations between muscle NDUFB6 mRNA and protein levels in muscle biopsies from 8 young and 8 elderly twins before (D) and $\operatorname{after}(\mathrm{E})$ insulin stimulation. Results are mean \pm SEM. ${ }^{*} P<0.05$. human muscle (regression coefficient, $0.1 ; P<0.0001$ ). This regression coefficient indicates that when $P G C-1 \alpha$ mRNA increases by 1 unit, NDUFB6 expression increases by 0.1 unit.

The NDUFB6 gene is subject to DNA methylation. DNA methylation is a potential mechanism by which expression of a gene may be regulated. Cytosine residues occurring in CG dinucleotides are targets for DNA methylation, and gene expression is usually reduced when DNA methylation takes place at a promoter. We therefore searched for polymorphisms in the promoter region of NDUFB6 that would introduce DNA methylation sites and putative transcription factor-binding sites using the GEMS Launcher
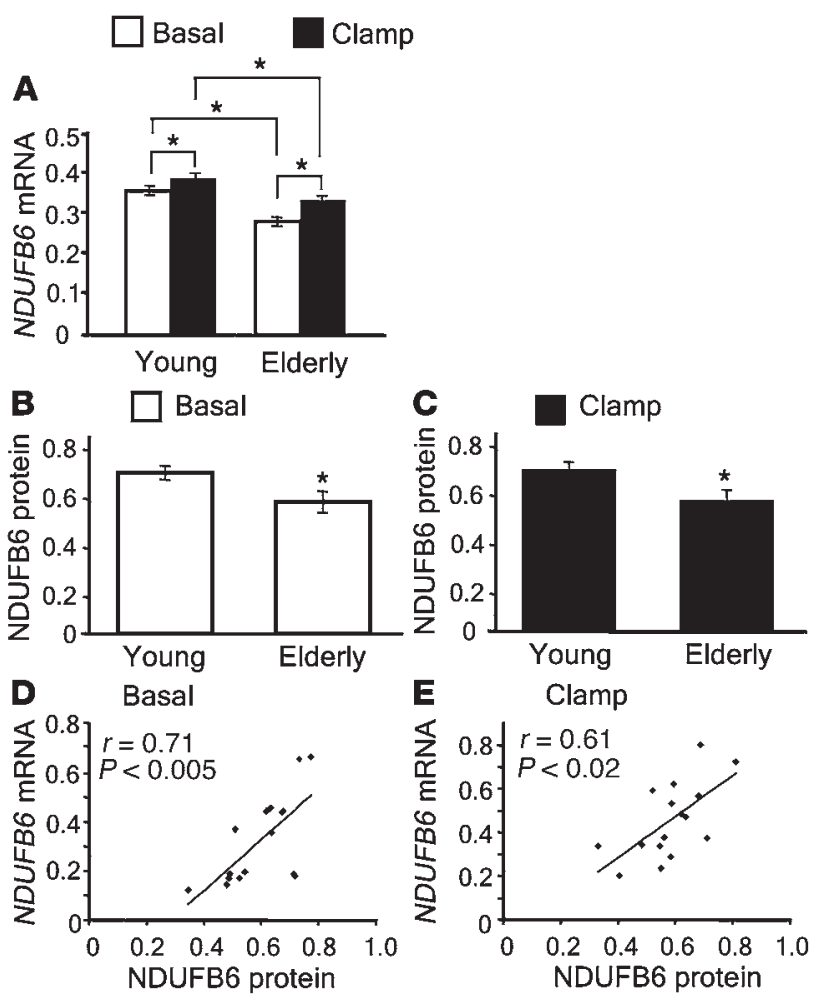


\section{Table 2}

Best-fitting biometric models for NDUFB6 expression in muscle from 91 young and 70 elderly twins during clamp

\begin{tabular}{lccccc} 
& \multicolumn{5}{c}{ Components of variance $^{\mathrm{A}}$} \\
Model & $\begin{array}{c}\text { Additive } \\
\text { genetic }\end{array}$ & $\begin{array}{c}\text { Genetic } \\
\text { dominance }\end{array}$ & $\begin{array}{c}\text { Common } \\
\text { environment }\end{array}$ & $\begin{array}{c}\text { Unique } \\
\text { environment }\end{array}$ \\
Young & AE & $0.65(0.36-0.82)$ & - & - & $0.35(0.18-0.64)$ \\
Elderly & $A E$ & $0.52(0.14-0.75)$ & - & - & $0.48(0.25-0.86)$
\end{tabular}

Data are presented as proportion of total variance with $95 \%$ confidence intervals. $\chi^{2}=0.00, P=1.00$, and Akiake information criteria $=-2.00$ for the goodness-of-fit tests for NDUFB6 expression during clamp in both young and elderly twins. AValues denote proportion of variance attributed to the respective component.

(version 4.1; Genomatix). A polymorphism, rs629566 (A/G), introduced a potential DNA methylation site by changing the sequence $C A$ to $C G$ at position -544 (Figure 2 C). In addition, the polymorphism resides in a putative transcription factor-binding site. Of the studied subjects, $54.9 \%$ carried the $A / A$ genotype, $35.2 \%$ carried the $A / G$ genotype, and $9.9 \%$ carried the $G / G$ genotype of rs629566. Young twins with the rs629566 G/G genotype $(n=6)$ had greater relative NDUFB6 mRNA expression in muscle compared with young twins carrying the $A / G(n=28)$ or $A / A(n=57)$ genotypes $(G / G, 0.51 \pm 0.051$; versus $A / G, 0.39 \pm 0.024$; and $A / A$, $0.39 \pm 0.017 ; P<0.05 ;$ Figure $2 A)$. In contrast, elderly twins carrying the rs629566 $\mathrm{G} / \mathrm{G}$ genotype $(n=9)$ had lower relative muscle NDUFB 6 mRNA levels than did carriers of the $A / G(n=29)$ or $A / A$ $(n=32)$ genotypes $(G / G, 0.24 \pm 0.033$; versus $A / G, 0.33 \pm 0.018$; and $A / A 0.36 \pm 0.017 ; P<0.05$; Figure $2 \mathrm{~B})$.

There were 3 additional methylation target sites close to rs629566 (position -544) in the NDUFB6 promoter, at positions -634, -663, and -676 (Figure 2C). We assessed the degree of DNA methylation of these 4 target sites using bisulfite genomic sequencing in young and elderly twins carrying the rs629566 G/G genotype. Interestingly, these sites were only methylated in elderly carriers of the rs629566 $G / G$ genotype, in whom $58 \% \pm 16 \%$ of the sites were methylated. In contrast, none of the young twins with this genotype showed methylation of these sites. Twins carrying the rs629566 A/A genotype were used as controls, and the NDUFB6 $-545 C$ nucleotide was not methylated in any of the $A / A$ carriers. Furthermore, the degree of DNA methylation of these target sites was negatively correlated with the level of basal $(r=-0.61 ; P<0.05 ; n=14)$ and insulin-

\section{Figure 2}

Association among the NDUFB6 polymorphism rs629566 (A/G), DNA methylation, and mRNA expression in muscle. (A and $\mathbf{B})$ Association between skeletal muscle NDUFB6 mRNA levels and rs629566 (A/G) in young $(\mathbf{A} ; n=91)$ and elderly $(\mathbf{B} ; n=70)$ twins after a hyperinsulinemic clamp. The level of NDUFB6 transcripts were normalized to the mRNA level of endogenous cyclophilin A, and the NDUFB6/cyclophilin $A$ ratio was calculated for each sample. In order to adjust for the lack of independence between monozygotic and dizygotic twins, all mean differences between groups were compared using GEE methodology. Results are mean \pm SEM. ${ }^{*} P<0.05$. (C) The NDUFB6 promoter sequence investigated, showing rs629566 (-544) and 3 additional DNA methylation target sites: $-634,-663$ and -676 . (D and E) Correlation between percent DNA methylation and basal (D) and insulinstimulated (E) NDUFB6 expression in muscle $(n=14$; correlations adjusted for age). stimulated $(r=-0.62 ; P<0.05 ; n=14)$ muscle NDUFB6 mRNA expression (Figure 2, D and E), suggesting that the degree of DNA methylation of the NDUFB6 promoter influences the age-related reduction in NDUFB6 expression.

Age does not affect DNA methylation in the PGC-1 $\alpha$ and UQCRB genes. To determine whether the age-related increase in DNA methylation of the NDUFB6 promoter is a universal aging phenomenon, we measured the level of DNA methylation in the promoter of 2 additional genes, UQCRB (from complex 3 ) and $P G C-1 \alpha$, in muscle from a subset of young and elderly twins. The level of DNA methylation was related to gene expression. UQCRB and PGC- $1 \alpha$ are among the genes that show reduced expression in diabetic muscle, and we have previously shown that $P G C-1 \alpha$ expression declines with age $(3,5)$. The relative $m R N A$ level of $U Q C R B$ was also reduced in muscle from elderly compared with young twins $(0.46 \pm 0.02$ versus $0.64 \pm 0.03 ; P<0.0005)$. In contrast to the observations of NDUFB6, there was no difference in the level of DNA methylation of the $U Q C R B$ promoter between young and elderly twins (young, $0.36 \% \pm 0.2 \%, n=8$; elderly, $0.26 \% \pm 0.2 \%, n=8 ; P=0.6$ ). It is of particular note that the degree of DNA methylation was very low in both age groups. No methylation of the UQCRB promoter was found in 6 of 8 elderly and 5 of 8 young subjects. There was no significant correlation between the level of DNA methylation and mRNA expression of the UQCRB gene in skeletal muscle $(r=0.36$; $P=0.2)$. Neither was there any age-related difference in DNA methylation of the $P G C-1 \alpha$ promoter (young, $12.4 \% \pm 3.3 \%, n=10$; elderly, 9.6\% $\pm 3.4 \%, n=9 ; P=0.6$ ), and no correlation was found between the degree of DNA methylation and $P G C-1 \alpha$ expression in muscle $(r=0.15 ; P=0.5)$. These results demonstrate that DNA methylation is associated with an age-related decline in gene expression in human muscle in some, but not all, genes.
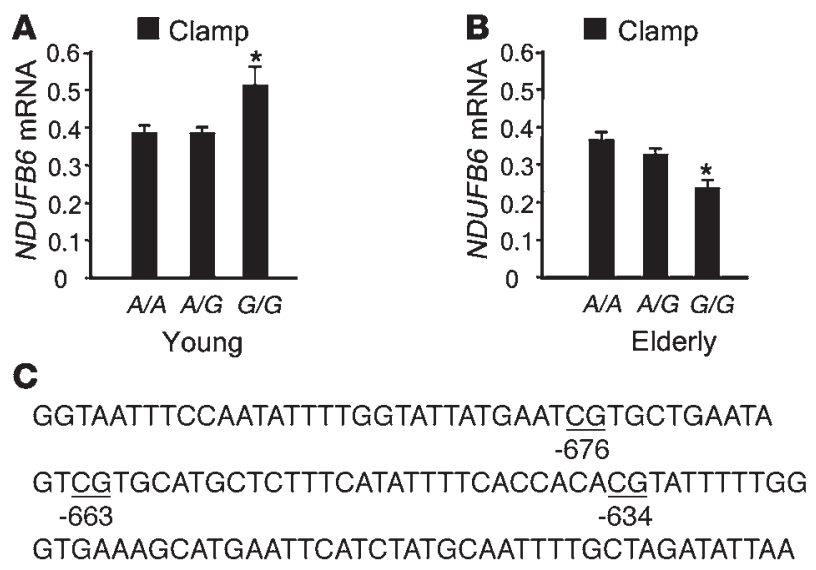

GCTACTCTTTTCACTATTCCTGTACAGATGGAGGGAC[A/G]G
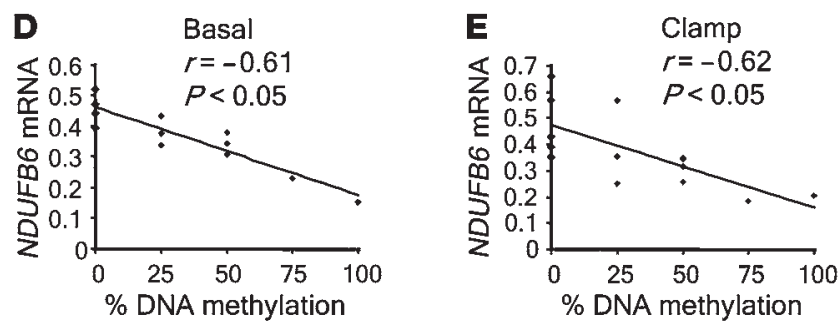
Table 3

Association between tagSNPs in the NDUFB6 gene and insulin-stimulated NDUFB6 mRNA expression in young and elderly twins

\begin{tabular}{|c|c|c|c|c|c|}
\hline Young & 11 & 12 & 22 & $P$ & Corrected $P$ \\
\hline rs2376223 & $0.42 \pm 0.02(53)$ & $0.39 \pm 0.02(29)$ & $0.35 \pm 0.05(6)$ & NS & NS \\
\hline rs540467 & $0.41 \pm 0.015(63)$ & $0.38 \pm 0.028(21)$ & $0.36 \pm 0.07(3)$ & NS & NS \\
\hline rs2050456 & $0.42 \pm 0.016(56)$ & $0.35 \pm 0.02(31)$ & $0.36 \pm 0.08(2)$ & NS & NS \\
\hline rs12003093 & $0.41 \pm 0.017(53)$ & $0.38 \pm 0.02(31)$ & $0.36 \pm 0.07(3)$ & NS & NS \\
\hline rs829535 & $0.39 \pm 0.017(53)$ & $0.39 \pm 0.022(32)$ & $0.46 \pm 0.06(4)$ & NS & NS \\
\hline rs653790 & $0.39 \pm 0.016(59)$ & $0.38 \pm 0.024(26)$ & $0.56 \pm 0.06(4)$ & NS & NS \\
\hline rs629566 & $0.39 \pm 0.017(57)$ & $0.39 \pm 0.024(26)$ & $0.51 \pm 0.051(6)$ & 0.026 & \\
\hline rs17218354 & $0.40 \pm 0.018(40)$ & $0.38 \pm 0.02(32)$ & $0.41 \pm 0.03(15)$ & NS & NS \\
\hline rs10813831 & $0.40 \pm 0.016(57)$ & $0.39 \pm 0.026(24)$ & $0.49 \pm 0.05(5)$ & 0.04 & NS \\
\hline rs3824456 & $0.40 \pm 0.016(57)$ & $0.38 \pm 0.02(30)$ & $0.53 \pm 0.09(2)$ & NS & NS \\
\hline rs4013845 & $0.39 \pm 0.02(36)$ & $0.39 \pm 0.02(32)$ & $0.42 \pm 0.03(16)$ & NS & NS \\
\hline Elderly & 11 & 12 & 22 & $P$ & Corrected $P$ \\
\hline rs2376223 & $0.35 \pm 0.018(33)$ & $0.30 \pm 0.018(31)$ & $0.40 \pm 0.045(5)$ & NS & NS \\
\hline rs540467 & $0.35 \pm 0.018(34)$ & $0.31 \pm 0.019(29)$ & $0.34 \pm 0.06(3)$ & NS & NS \\
\hline rs2050456 & $0.34 \pm 0.016(43)$ & $0.30 \pm 0.02(22)$ & $0.41 \pm 0.05$ & NS & NS \\
\hline rs12003093 & $0.35 \pm 0.018(32)$ & $0.32 \pm 0.02(27)$ & $0.29 \pm 0.05(5)$ & NS & NS \\
\hline rs829535 & $0.33 \pm 0.016(43)$ & $0.32 \pm 0.021(24)$ & $0.54 \pm 0.05(1)$ & NS & NS \\
\hline rs653790 & $0.37 \pm 0.016(34)$ & $0.31 \pm 0.018(28)$ & $0.22 \pm 0.04(7)$ & 0.002 & 0.024 \\
\hline rs629566 & $0.36 \pm 0.017(32)$ & $0.33 \pm 0.018(29)$ & $0.24 \pm 0.033(9)$ & 0.005 & \\
\hline rs17218354 & $0.30 \pm 0.02(21)$ & $0.35 \pm 0.019(29)$ & $0.33 \pm 0.03(16)$ & NS & NS \\
\hline rs10813831 & $0.36 \pm 0.017(32)$ & $0.32 \pm 0.019(25)$ & $0.24 \pm 0.03(11)$ & 0.002 & 0.024 \\
\hline rs3824456 & $0.31 \pm 0.015(47)$ & $0.35 \pm 0.025(16)$ & $0.44 \pm 0.04(6)$ & 0.02 & NS \\
\hline rs4013845 & $0.30 \pm 0.03(18)$ & $0.37 \pm 0.025(19)$ & $0.34 \pm 0.03(16)$ & NS & NS \\
\hline All & MAF & HET & HW $P$ & & \\
\hline rs2376223 & 0.267 & 0.396 & 1.0 & & \\
\hline rs540467 & 0.209 & 0.332 & 1.0 & & \\
\hline rs2050456 & 0.208 & 0.353 & 0.48 & & \\
\hline rs12003093 & 0.258 & 0.407 & 0.56 & & \\
\hline rs829535 & 0.206 & 0.36 & 0.27 & & \\
\hline rs653790 & 0.25 & 0.353 & 0.50 & & \\
\hline rs629566 & 0.26 & 0.335 & 0.09 & & \\
\hline rs17218354 & 0.47 & 0.408 & 0.018 & & \\
\hline rs10813831 & 0.272 & 0.339 & 0.073 & & \\
\hline rs3824456 & 0.224 & 0.311 & 0.2 & & \\
\hline rs4013845 & 0.466 & 0.377 & 0.003 & & \\
\hline
\end{tabular}

Results are mean \pm SEM, with $n$ shown in parentheses; 1 , major allele; 2 , minor allele. In order to adjust for the lack of independence between monozygotic and dizygotic twins, all comparisons of mean differences between groups were performed using GEE methodology. Corrected $P$ values were corrected for multiple testing based on the number of NDUFB6 tagSNPs genotyped in the twins. The assay did not work in the rs920029 plex. HET, observed heterozygosity; HW, Hardy-Weinberg; MAF, minor allele frequency.

Other polymorphisms in the NDUFB6 gene are also associated with gene expression in buman muscle. In order to establish whether other polymorphisms are associated with the level of NDUFB6 expression in muscle, 12 tagSNPs (see Methods) from the NDUFB6 locus were selected using genotype data from the International HapMap project (4) together with the Tagger program $\left(r^{2}>0.8\right)$ (10) (Table 3). Two additional SNPs, rs653790 and rs10813831, were significantly associated with NDUFB6 expression in muscle of elderly twins $(P=0.024$; Table 3$)$. These 2 SNPs were in strong linkage disequilibrium (LD) with rs629566 (rs653790 and rs629566, $\mathrm{D}^{\prime}=1$, $r^{2}=0.90 ;$ rs10813831 and rs629566, $\mathrm{D}^{\prime}=0.97, r^{2}=0.89$; Figure 3).

Association of common variants of the NDUFB6 gene with the risk of T2DM. We next investigated whether the polymorphism rs629566, which introduces the DNA methylation site, is associated with risk of T2DM in a screening set composed of 751 cases with T2DM and 715 healthy controls from the Botnia cohort. The clinical characteristics of these subjects are described in
Table 1. We found no significant difference between the frequency of the GG genotype in cases (5.2\%) and controls $(6.3 \%$; $P=0.58)$. We therefore extended the study to examine whether other common variants of the NDUFB6 gene are associated with increased risk of T2DM. First, 12 tagSNPs covering the majority of the genetic variation in NDUFB6 were selected using HapMap data from the CEU cohort (see Methods) and genotyped in the screening set (Table 1 and Supplemental Table 1; supplemental material available online with this article; doi:10.1172/ JCI30938DS1). The LD structure of this screening set showed that most of the genetic variation $\left(\mathrm{D}^{\prime}>0.8\right)$ in NDUFB6 in our cohort was covered with 3 tagSNPs: rs540467, rs2050456, and rs629566 (Figure 3). Although carriers of the rs540467 A/A genotype had a nominally increased risk of T2DM compared with $G / G$ and $G / A$ carriers, with an odds ratio (OR) of 2.06 (95\% confidence interval, 1.07-3.98; $P=0.03)$, when logistic regression was performed with adjustments for BMI, gender, and age 


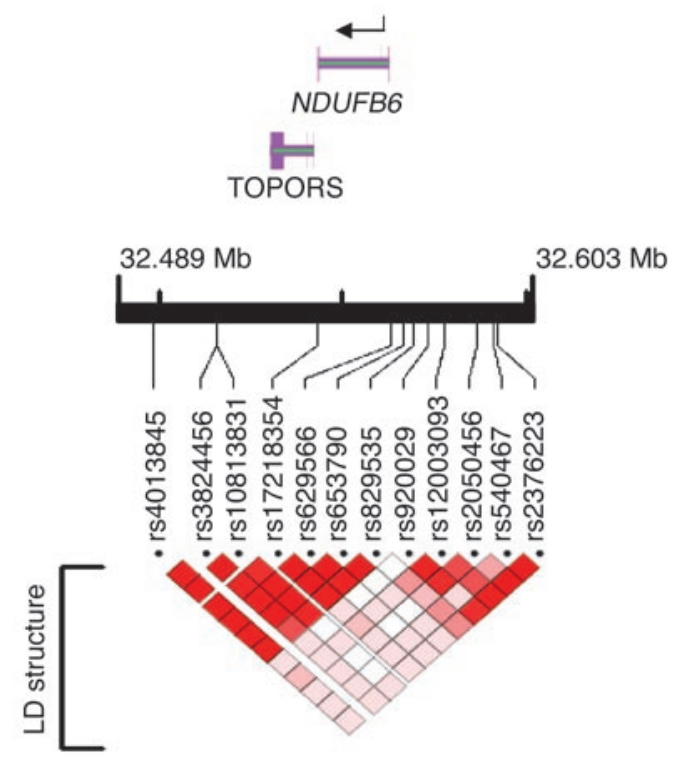

at onset (cases) or age at visit (controls) (Supplemental Table 1), this result would not stand correction for multiple testing. Second, the 3 tagSNPs (rs540467, rs2050456, and rs629566) that covered most of the genetic variation in NDUFB 6 in the Botnia cohort were then genotyped in a larger replication set, consisting of 2,830 cases and 3,740 healthy controls from the Malmö cohort (Table 1 and Supplemental Table 2). Also in this replication set, carriers of the rs540467 $A / A$ genotype showed increased risk of T2DM compared with carriers of the $G / G$ and $G / A$ genotype, with an OR of 1.40 (95\% confidence interval, 1.05-1.87; $P=0.02$; Supplemental Table 2). Because the LD structure was quite similar and a heterogeneity test excluded a significant difference between the Botnia and the Malmö cohorts, we also performed a combined analysis, yielding an OR of 1.46 (95\% confidence interval, 1.13-1.90; $P=0.0038$ ) for the rs540467 $\mathrm{A} / \mathrm{A}$ genotype. Analyzing the data using Mantel-Haenszel test gave virtually the same result (OR, 1.41; 95\% confidence interval, $1.12-1.77 ; P=0.0038$ ). We used the MODEL program (http:// pngu.mgh.harvard.edu/ purcell/model/) to search for the best genetic model, and a recessive model was suggested to best explain the effect of rs540467 on T2DM risk.

Effect of NDUFB6 on $\mathrm{VO}_{2}$ max and glucose uptake in twins. Mitochondrial function and oxidative capacity influence $\mathrm{VO}_{2}$ max and glucose uptake. Both $\mathrm{VO}_{2} \max$ and insulin-stimulated glucose uptake usually decline with age, but it is not known whether they are affected by NDUFB6. We therefore examined whether muscle NDUFB 6 mRNA expression was associated with $\mathrm{VO}_{2}$ max and insulin-stimulated glucose uptake, using multivariate regression analysis including age, sex, and BMI as covariates (Supplemental Table 3). Basal NDUFB6 expression was positively related to both $\mathrm{VO}_{2} \max$ (regression coefficient, 10.9; $P<0.005$ ) and insulin-stimulated glucose uptake (regression coefficient, 6.2; $P<0.003$ ). We further tested whether 2 NDUFB6 polymorphisms, rs629566 and rs540467, were associated with $\mathrm{VO}_{2}$ max and insulin-stimulated glucose uptake (Table 4). Our data suggest that rs629566 may be associated with $\mathrm{VO}_{2}$ max and insulin-stimulated glucose uptake in the elderly twins. It should be noted that this post-hoc test was not corrected for multiple comparisons.

\section{Figure 3}

Schematic presentation of the NDUFB6 gene with the analyzed tagSNPs and LD structure. LD between the NDUFB6 SNPs was analyzed using Haploview (50). D' values with $95 \%$ confidence intervals were calculated as a measure of LD (51). The analysis was performed based on the genotyping results from the Botnia cohort. The figure was prepared using LocusView 2.0 (http://www.broad.mit.edu/mpg/locusview/). The following SNPs were in LD: rs540467 and rs2376223 ( $\left.\mathrm{D}^{\prime}=1\right)$; rs2050456, rs12003093, and rs920029 ( $D^{\prime}>0.83$ ); and rs629566, rs829535, rs653790, rs17218354, rs10813831, rs3824456, and rs4013845 ( $\left.D^{\prime}>0.94\right)$.

\section{Discussion}

A set of nuclear-encoded OXPHOS genes show reduced expression in muscle from patients with T2DM; however, it remains unknown whether this is an inherited or acquired defect (3). We therefore attempted to determine which mechanisms regulate OXPHOS gene expression in human muscle. A nuclear-encoded gene, NDUFB6 (from complex 1 in the respiratory chain), was selected for this analysis because its expression is significantly reduced in diabetic muscle, the NDUFB6 gene is located under a linkage peak for T2DM on chromosome 9, and complex 1 is suggested to be rate limiting for NADH oxidation $(3,9,11,12)$. The key finding of the present study was that genetic (polymorphism), epigenetic (DNA methylation), and nongenetic (e.g., age) factors were associated with the expression level of NDUFB6 in human skeletal muscle.

Insulin resistance in skeletal muscle is a major contributor to the increased prevalence of T2DM with age, and oxidative capacity in muscle is a good predictor of insulin sensitivity (13). To determine why insulin sensitivity decreases with age, we analyzed the expression level of NDUFB6 in muscle biopsies from young and elderly nondiabetic twins. Elderly twins had $15 \%-22 \%$ lower levels of NDUFB6 than did young twins. This decline was of the same magnitude as the $19 \%$ decrease previously observed in muscle from patients with T2DM (3). An age-related reduction in expression of OXPHOS genes is likely to reduce mitochondrial function and insulin action in muscle and eventually increase the risk of developing T2DM. In support of this hypothesis, aging has been associated with decreased OXPHOS expression and mitochondrial oxidative capacity in muscle $(2,14)$. Furthermore, healthy elderly individuals show increased fat accumulation, reduced mitochondrial activity, and reduced insulin-stimulated glucose uptake in skeletal muscle compared with young subjects (15). In this study the level of NDUFB6 in muscle was positively associated with glucose uptake and $\mathrm{VO}_{2}$ max. This is consistent with our previous findings that expression of NADH dehydrogenase 1 , another gene from the first respiratory complex, is related to glucose flux in muscle (16).

Not all individuals respond identically to an affluent diabetogenic environment. Several studies provide evidence that genetic factors are important contributors to the large interindividual variation in diabetes susceptibility $(6,17-20)$. To address this issue, we estimated heritability for muscle expression of NDUFB6 in young and elderly twins. Heritability was $65 \%$ and $52 \%$ in young and elderly twins, respectively, suggesting that expression of NDUFB 6 in muscle is under genetic control. Consistent with this, insulin-resistant offspring of patients with T2DM have previously been reported to have impaired mitochondrial function in muscle $(1,21,22)$. 


\section{Table 4}

Association between the NDUFB6 polymorphisms rs629566 and rs540467 and $\mathrm{VO}_{2} \mathrm{max}$ and glucose uptake

\begin{tabular}{|c|c|c|c|c|}
\hline rs629566 & $A / A$ & $A / G$ & $G / G$ & $P$ \\
\hline \multicolumn{5}{|l|}{ Young } \\
\hline $\mathrm{VO}_{2} \max ^{\mathrm{A}}$ & $39 \pm 0.9(63)$ & $39 \pm 1.3(33)$ & $42 \pm 2.6(9)$ & NS \\
\hline Glucose uptake clampB & $11.50 \pm 0.42(61)$ & $11.69 \pm 0.56(34)$ & $13.35 \pm 1.08(9)$ & NS \\
\hline \multicolumn{5}{|l|}{ Elderly } \\
\hline $\mathrm{VO}_{2} \mathrm{max}^{\mathrm{A}}$ & $29 \pm 1.0(38)$ & $24 \pm 1.1(35)$ & $24 \pm 2.2(9)$ & $<0.05^{C}$ \\
\hline Glucose uptake clampB & $10.8 \pm 0.5(40)$ & $8.8 \pm 0.5(37)$ & $9.9 \pm 1.1(9)$ & $<0.05^{c}$ \\
\hline rs540467 & $G / G$ & $G / A$ & $A / A$ & $P$ \\
\hline \multicolumn{5}{|l|}{ Young } \\
\hline $\mathrm{VO}_{2} \max ^{\mathrm{A}}$ & $39.4 \pm 0.9(70)$ & $37.5 \pm 1.4(29)$ & $50.3 \pm 4.3(3)$ & $<0.05^{D}$ \\
\hline Glucose uptake clampB & $12.1 \pm 0.4(71)$ & $10.7 \pm 0.6(27)$ & $13.5 \pm 1.9(3)$ & NS \\
\hline \multicolumn{5}{|l|}{ Elderly } \\
\hline $\mathrm{VO}_{2} \max ^{\mathrm{A}}$ & $26.2 \pm 1.0(43)$ & $27.2 \pm 1.2(31)$ & $23.2 \pm 3.4(4)$ & NS \\
\hline Glucose uptake clampB & $10.1 \pm 0.5(44)$ & $10.4 \pm 0.6(32)$ & $7.1 \pm 1.5(5)$ & $0.05^{\mathrm{D}}$ \\
\hline
\end{tabular}

Data are expressed as mean \pm SEM, with $n$ shown in parentheses. In order to adjust for the lack of independence between monozygotic and dizygotic twins, all comparisons of mean differences between groups were performed using GEE methodology. AShown in $\mathrm{ml} / \mathrm{kg} / \mathrm{min}$. BShown in $\mathrm{mg} / \mathrm{kg}$ lean body mass $/ \mathrm{min}$. ${ }^{\mathrm{C}} A / A$ vs. $A / G+G / G$. ${ }^{D} G / G+G / A$ vs. $A / A$. $P$ values were not adjusted for multiple testing.

did not show any appreciable increase in DNA methylation with age, suggesting that factors other than DNA methylation must contribute to the observed age-related decline in PGC-1 $\alpha$ expression in human skeletal muscle. Indeed, we have previously shown that a common Gly482Ser polymorphism in the PGC- $1 \alpha$ gene is associated with gene expression (5). Neither did we observe any age-related increase in DNA methylation of another OXPHOS gene, UQCRB, whose expression decreased with aging. Collectively, these data suggest that DNA methylation does not explain all the age-related decline in OXPHOS gene expression in muscle.

Because a previous study suggested that NDUFB6 may play a role in the pathogenesis of T2DM (3), and because SNPs in the NDUFB6 gene were associated with the expression levels in skeletal muscle, we investigated whether common variants in the NDUFB6 gene also confer to a risk of developing T2DM. Although one com-

To gain further insight into the genetic regulation of NDUFB6 expression in muscle, we investigated whether allelic variation in this gene is associated with mRNA level. We identified a polymorphism in the promoter region of NDUFB6, rs629566 (A/G), which, by changing a CA dinucleotide to CG, introduces a DNA methylation site. This SNP could also create a putative, as yet unvalidated, transcription factor-binding site. While young twins carrying the rs629566 G/G genotype had significantly greater NDUFB6 expression in muscle than did young carriers of the $A / A$ or $A / G$ genotype, the same genotype was somewhat paradoxically associated with reduced expression in muscle from elderly twins. Interestingly, the rs629566 DNA methylation site described above as well as 3 adjacent methylation sites were only methylated in elderly twins, not young twins, carrying the NDUFB6 rs629566 G/G genotype. When DNA methylation takes place at a promoter, gene expression is usually reduced. Indeed, there was a significant negative correlation between the degree of DNA methylation and the mRNA level of NDUFB 6 in muscle. Our data demonstrate that combinations of genetic (polymorphism) and epigenetic (DNA methylation) factors are associated with the age-related decline in gene expression. To the best of our knowledge, this is the first demonstration of how changes in DNA methylation during postnatal life may play a role in the pathogenesis of insulin resistance. This also opens up the possibility that epigenetic factors such as DNA methylation and histone acetylation could predispose an individual to insulin resistance and T2DM. In addition, 2 tagSNPs, rs653790 and rs10813831, which are in strong LD with rs629566, were also significantly associated with NDUFB6 mRNA levels in skeletal muscle. However, not all tagSNPs in the block were associated with gene expression. The most plausible reason for this is the lack of statistical power, as this study included only 196 twins.

We extended the previous observation in rodents (3) that the transcriptional coactivator PGC-1 $\alpha$ is also associated with NDUFB6 expression in human skeletal muscle, where the expression levels of PGC-1 $\alpha$ and NDUFB6 were positively related $(P<0.0001)$. However, in contrast to the situation with NDUFB6, the $P G C-1 \alpha$ gene mon SNP (rs540467) in the NDUFB6 gene was associated with a nominally increased risk of T2DM, the evidence for a genetic association between SNPs in this gene and T2DM was weak in the present study. Given the large number of tests performed, our study was not sufficiently powerful to detect subtle effects of these variants on risk of developing T2DM.

Several research groups, including our own, have recently performed genome-wide association scans (GWASs) in order to identify common polymorphisms associated with increased risk of T2DM (23-27). Collectively, these 5 scans identified at least 5 new SNPs and/or loci significantly associated with T2DM. The NDUFB6 locus was not among these 5 genes and loci, but it should be kept in mind that this first analysis was only based upon replication studies of 107 SNPs with the strongest association with T2DM. It will be very difficult to exclude any SNP among the top $1 \%$ (which includes more than 4,000 SNPs on the Affymetrix $500 \mathrm{~K}$ DNA chip). We therefore looked carefully at the NDUFB6 locus in 3 GWASs, whose authors shared their data prior to publication (DGI, ref. 23; WTCCC/UK, ref. 25; FUSION, ref. 26). While the DGI and WTCCC/UK studies used the Affymetrix 500K DNA chip for GWAS $(23,25)$, FUSION used the Illumina HumanHap300 BeadChip technology (26). A total of 23 SNPs in the NDUFB6 region were analyzed in the scans, 5 of them found in all studies. Two polymorphisms were associated with increased risk of the disease in the GWAS by FUSION, rs11795343 (OR, 1.69; 95\% confidence interval, 1.32-2.16; $P=0.00002$ ) and rs659527 (OR, 1.36; $95 \%$ confidence interval, $1.13-1.63 ; P=0.001$ ) for dominant models (26). These SNPs were localized in the genomic region examined in the present study (see Methods). Because these 2 SNPs were not in the HapMap when we selected tagSNPs, they were not selected for genotyping in the present study, nor were they on the Affymetrix 500K DNA chip. Moreover, 6 SNPs from the 3 studies showed association to T2DM with nominal $P$ values of 0.01-0.05: rs920029, rs629566, rs628425, rs669260, rs3824456, and rs10971037. It should be kept in mind that a SNP that is subject to methylation (i.e., rs629566) might not be easy to detect in an 
association study, given the change in risk dependent upon degree of methylation and whether risk or nonrisk alleles are methylated. The SNP rs540467 was not present on the Affymetrix chip, nor was it significantly associated with T2DM in the FUSION study. Collectively, the WGASs do not exclude a role for variation in the NDUFB6 gene in the pathogenesis of T2DM, but further work that captures the whole genetic variation at the locus in large studies will be needed to confirm this.

NDUFB6 is one of several nuclear-encoded OXPHOS genes that show reduced expression in skeletal muscle from patients with T2DM (3). Therefore, genetic variation in additional OXPHOS genes may confer a risk of T2DM and thereby interact with NDUFB6 to influence the susceptibility to the disease. Furthermore, genetic, epigenetic, and nongenetic factors might also be associated with the expression level of other nuclear-encoded OXPHOS genes. This matter needs to be addressed in future research. Several other studies have investigated the mechanisms by which OXPHOS and mitochondrial function decline during aging and with T2DM. Diminished mitochondrial content and mitochondrial copy number are believed to influence OXPHOS in diabetic muscle $(22,28-30)$. Oxidative stress, mitochondrial DNA mutations, and apoptosis are other mechanisms suggested to influence mitochondrial function and OXPHOS expression during aging $(31,32)$. Furthermore, inflammation induced by FFAs and cytokines leading to oxidative stress also affects mitochondrial biogenesis in skeletal muscle (33).

In summary, the present study demonstrates that the interaction of genetic and epigenetic factors contributes to the agerelated decline in expression of the OXPHOS gene, NDUFB6, in human muscle. Our results provide an example of how genetic and epigenetic factors may interact to confer an age-dependent susceptibility to insulin resistance.

\section{Methods}

\section{Subjects}

Twins. Subjects were identified from the Danish Twin Register $(20,34)$. Selection criteria for the young and elderly twins were described previously (5), and the participants' characteristics are described in Table 1.

Botnia cohort (screening set). We studied 1,466 unrelated individuals from the Botnia study, 751 of whom were diagnosed with T2DM at more than 35 years of age, and 715 of whom were nondiabetic controls with no firstdegree relatives with T2DM (Table 1). The Botnia study is a family-based study established in 1990 that aims to identify T2DM susceptibility genes $(35,36)$. Subjects were classified into different stages of glucose tolerance based on an oral glucose tolerance test. Individuals with genetically verified maturity-onset diabetes of the young or type 1 diabetes were excluded from the study.

Malmö cohort (replication set). This included 2,830 cases with T2DM from a local diabetes registry (37) and 3,740 unrelated, ethnically matched healthy control individuals from the Malmö Diet and Cancer Study (38) (Table 1). T2DM was diagnosed according to WHO criteria (39), with C-peptide concentrations of at least $0.3 \mathrm{nmol} / \mathrm{l}$, no GAD antibodies, and age at onset of 35 years or more. The control individuals had a level of fasting blood glucose of less than $5.6 \mathrm{mmol} / \mathrm{l}$ and no known family history of T2DM (Table 1).

All the present studies were approved by the regional Ethical Committees of Southern Denmark (Odense, Denmark), Vaasa Central Hospital (Vaasa, Finland), Lund University (Lund, Sweden), and Helsinki University (Helsinki, Finland) and were conducted in accordance with the Helsinki Declaration.

\section{Clinical examination and muscle biopsies from twins}

Twins underwent a 2-day clinical examination, including a 2-hour hyperinsulinemic euglycemic clamp $\left(40 \mathrm{mU} / \mathrm{m}^{2} / \mathrm{min}\right)$ combined with indirect calorimetry during the basal and insulin-stimulated steady-state periods, as previously described $(5,20)$. Metabolic rates (i.e. glucose disposal, glucose and fat oxidation, and nonoxidative glucose metabolism) were calculated as previously reported and expressed as $\mathrm{mg} / \mathrm{kg}$ lean body mass $/ \mathrm{min}$ $(20,40)$. Basal and insulin-stimulated muscle biopsies were obtained from the vastus lateralis muscle under local anesthesia. Plasma glucose and insulin concentrations and specific activity of tritiated water were measured as previously described $(34,41,42)$.

\section{Analysis of NDUFB6 and UQCRB mRNA levels in skeletal muscle}

NDUFB6 and UQCRB mRNA levels were quantified using TaqMan RealTime PCR with an ABI 7900 system (Applied Biosystems) using gene-specific probes and primer pairs for NDUFB6 (Assays-on-Demand, Hs00159583_m1; Applied Biosystems) and UQCRB (Assays-on-Demand, Hs00559884_m1; Applied Biosystems). The transcript quantity was normalized to the mRNA level of cyclophilin A (4326316E; Applied Biosystems).

\section{Analysis of NDUFB6 protein levels in skeletal muscle by Western blot} Protein lysate was prepared from muscle biopsies as previously described (43). Lysate proteins $(20 \mu \mathrm{g})$ were separated using Criterion $10.5 \%-14 \%$ Tris$\mathrm{HCl}$ linear gradient gels (Invitrogen), and then transferred to PVDF membranes (Invitrogen). After blocking (Tris-buffered saline, 0.1\% Tween-20, and $5 \%$ nonfat dry milk) for 1 hour, the membranes were incubated with primary antibodies for NDUFB6 $(15.5 \mathrm{kDa}$, stock $1 \mu \mathrm{g} / \mu \mathrm{l}$, dilution 1:1000, A21359; Invitrogen) overnight at $4{ }^{\circ} \mathrm{C}$ followed by incubation with secondary antibodies linked to horseradish peroxidase, antimouse (stock $1 \mu \mathrm{g} / \mu \mathrm{l}$, dilution 1:100 000, Pierce Biotechnology Inc.). Immunoreactive proteins were visualized by chemiluminescence using SuperSignal West Dura Extended Duration Substrate (Pierce Biotechnology Inc.) and the Fujifilm Luminescent Image Analyzer LAS-3000 with a CCD camera (Fujifilm). Protein levels were then quantified using Multi Gauge software (Fujifilm).

\section{Genotyping}

Genomic DNA was extracted from blood by conventional methods (44). In the twins and the Malmö cohort, the rs629566 polymorphism of NDUFB6 was genotyped using an allelic discrimination assay (C_2734847_20; Applied Biosystems) performed with an ABI 7900 system (Applied Biosystems).

Genotype data from the International HapMap project (www.hapmap. org) (4) covering the NDUFB6 locus (chromosome 9, 32490000-32595000) from the CEU population was downloaded (data source, Rel 16c.1/phase 1 June 2005), and NDUFB6 tagSNPs - single nucleotide polymorphisms that could explain greater than $80 \%$ of the common variation $\left(r^{2}>0.8\right)$ at a selected locus and with a minor allele frequency greater than $5 \%$ - were selected using Tagger (10). Based on this analysis, the following NDUFB6 tagSNPs were selected and genotyped: rs2376223, rs540467, rs2050456, rs12003093, rs920029, rs829535, rs653790, rs629566, rs17218354, rs 10813831, rs3824456, and rs4013845. These SNPs were genotyped using a single-base extension method adopted for a MassArray (Sequenome) platform in the Profiling Polygenic Disease Swegene laboratory in Malmö.

A genotyping error rate of less than $1 \%$ determined by at least $6 \%$ regenotyping was used to secure genotyping accuracy. Native DNA was used for all genotyping except for the controls in the Malmö cohort, for which whole genome amplified DNA (deCODE Genetics) was used.

\section{DNA methylation}

Genomic DNA was isolated from muscle biopsies at the same time as RNA using the Tri Reagent kit, following the manufacturer's instruc- 
tions (Sigma-Aldrich). DNA bisulfite modification was accomplished using the EZ DNA methylation kit (Zymo Research). Bisulfite-modified DNA was amplified with primers designed using the previously described MethPrimer program (45).

NDUFB6. The following primers were used to amplify the sequence shown in Figure 2C: forward, 5 -GTTGTTTTTTGATTGTTGTATTATAATTTA-3'; reverse, 5'-AAAATACCCTAAACAACTATCTCAT-3'. To obtain more PCR product, a second, seminested PCR was performed using the forward primer 5'-TAAGTAGTTTTTTATTTAGGAATTTGGG-3' and the same reverse primer as above. The PCR product was then cloned into a TA vector (Fermentas), and 10 colonies of each reaction were purified as minipreps (Qiagen) and screened for correct insertion by restriction enzyme digestion with EcoRI, which cuts on both sides of the insert. The individual clones were sequenced, and the number of methylated sites was determined. We calculated the percentage of methylation for each individual as the total number of methylated sites in all clones analyzed divided by the total number of possible methylation sites in all clones analyzed.

UQCRB. A sequence located 239-62 base pairs upstream of the translation start (5'-TCTTCCCATCACATCACAACCAATACTTCTTAGAATGTAGCACGCAAAATGCCCCGCCTACTCCATTGGCCTCACCCTGGCCGAGAGGTGCTTACGCAGGTGCTGAACGGCAGTCGTCAGAACTGCGCCTGCGCAAGCGGCCTTTCTCTGTTCGCGATGTGACGTAACGCGCCTGCG-3', reverse complement) was chosen to analyze DNA methylation of the UQCRB promoter. This sequence contains a CPG island and 15 potential methylation sites. The method described above was used together with the following primer pair: forward, 5'-TTGGTTTTTTAATTTTTGATTTGTGT-3'; reverse, 5'-ACCTACTTACCAACCATTTTAACCA-3'. To obtain more PCR product, a second, seminested PCR was performed using the same forward primer as above and reverse primer 5'-AAAAACAAACTAAACCCAATCC-3'.

$P G C-1 \alpha$. A sequence located $961-770$ base pairs upstream of the translation start (5'-CGCTACATGTATGAAAAATAGGAGCCGGGAATCAAAGCTGATCTGAGCAGAGCAGCAGCGACTGTATTTACTAACACTTGTTTTCTGGGAGCCTATGAGAGAAATGGAAATAATTAGAAGGAAGCTGAAAGGATGGGGTTTTGTGGCTTGTTCTCCTTATATGGAGCAAAGAAAACTGCAGCAACTCTTCG$\left.3^{\prime}\right)$ was chosen to analyze DNA methylation of the $P G C-1 \alpha$ promoter. The method described above was used together with primer pair 1 (forward, 5'-TAGGGTATTAGGGTTGGAATTTAATG-3'; reverse, 5'-CCCATAACAATAAAAAATACCAACTC-3') and primer pair 2 (used for nested PCR; forward, 5'-TATTTTAAGGTAGTTAGGGAGGAAA-3'; reverse, 5'ATAACAATAAAAAATACCAACTCCC-3'.

\section{Statistics}

Generalized estimating equations and linear regression. Because of the strong intrapair correlation of twin data, conventional tests of differences between variable $(y)$ means are not valid. To correct for this dependence, we used generalized estimating equation (GEE) methodology $(y=\alpha+\beta x)$ to provide valid standard errors for the $\beta$ coefficients $(46,47)$. The $\beta$ coefficient was estimated from all observations, whereas for calculation of the variance of $\beta$, each twin pair was considered as 1 cluster. Clinical characteristics in Table 1 are presented as mean $\pm S D$, and all other results are presented as mean \pm SEM.

To identify factors independently associated with the response variable, we used backward-elimination multivariate regression analysis with $P>0.05$ as the threshold for exclusion of model terms. Also in this analysis we used GEE methodology to obtain valid tests. The regression coefficients presented correspond to linear regression coefficients.

Biometric modeling. The total phenotypic variance is the sum of the variance attributable to the effects of both genetic and environmental factors. The degree of genetic and environmental influence on a variable can be estimated using biometric modeling. Here, the models tested included the following contributions to variance: genetic variance due to additive genetic effects $\left(\mathrm{V}_{\mathrm{A}}\right)$, dominant genetic effects $\left(\mathrm{V}_{\mathrm{D}}\right)$, and environmental variance due to an individual environment not shared with co-twin $\left(\mathrm{V}_{\mathrm{E}}\right)$ or a common environment shared between co-twins $\left(\mathrm{V}_{\mathrm{C}}\right)$. Biometric modeling was conducted as previously described (48).

Association between genetic variation in the NDUFB6 gene and T2DM. The power to detect associations between SNPs in the NDUFB6 gene and T2DM in our case-control studies was calculated using the Genetic Power Calculator (49). With a minor allele frequency of 5\%, a T2DM frequency of $6 \%$, and a relative risk of 1.3 at $\alpha=0.05$, we have $38 \%$ power for a dominant model in the Botnia case-control study and a corresponding $93 \%$ power in the Malmö case-control study. To determine genotype association of individual SNPs with T2DM, we used a $\chi^{2}$ test. Two-tailed $P$ values are presented. Logistic regression was then applied for SNPs showing nominally significant association with T2DM, and this analysis was adjusted for BMI, sex, and age at onset (cases) or age at visit (controls). Allelic association, haplotype block structure, and LD structure were calculated using Haploview version 3.2 (50).

\section{Acknowledgments}

This investigation was supported by grants from Region Skåne, the Swedish Research Council, the Danish Medical Research Council, Danish Diabetes Association, Novo Nordisk, Malmö University Hospital, Swegene, the Diabetes Programme at Lund University, Hedlund, Bergvall, Wiberg, Påhlsson, Lundberg, and Exgenesis. We thank Margareta Svensson and Marianne Modest for their excellent technical assistance and Michael Boehnke for sharing data from the WGAS by FUSION (26).

Received for publication November 13, 2006, and accepted in revised form July 20, 2007.

Address correspondence to: Charlotte Ling, Department of Clinical Sciences, Diabetes and Endocrinology, Lund University, CRC, University Hospital Malmö, S-205 02 Malmö, Sweden. Phone: 46-40-391213; Fax: 46-40-391222; E-mail: charlotte.ling@med.lu.se.
1. Petersen, K.F., Dufour, S., Befroy, D., Garcia, R., and Shulman, G.I. 2004. Impaired mitochondrial activity in the insulin-resistant offspring of patients with type 2 diabetes. N. Engl.J. Med. 350:664-671.

2. Zahn, J.M., et al. 2006. Transcriptional profiling of aging in human muscle reveals a common aging signature. PLoS Genet. 2:e115.

3. Mootha, V.K., et al. 2003. PGC-1alpha-responsive genes involved in oxidative phosphorylation are coordinately downregulated in human diabetes. Nat. Genet. 34:267-273.

4. Patti, M.E., et al. 2003. Coordinated reduction of genes of oxidative metabolism in humans with insulin resistance and diabetes: Potential role of PGC1 and NRF1. Proc. Natl. Acad. Sci. U. S. A. 100:8466-8471.

5. Ling, C., et al. 2004. Multiple environmental and genetic factors influence skeletal muscle PGC-1alpha and PGC-1beta gene expression in twins. J. Clin. Invest. 114:1518-1526. doi:10.1172/ JCI200421889.

6. Ek, J., et al. 2001. Mutation analysis of peroxisome proliferator-activated receptor-gamma coactivator-1 (PGC-1) and relationships of identified amino acid polymorphisms to Type II diabetes mellitus. Diabetologia. 44:2220-2226.

7. Barroso, I., et al. 2006. Meta-analysis of the Gly482Ser variant in PPARGC1A in type 2 diabetes and related phenotypes. Diabetologia. 49:501-505.

8. Oberkofler, H., et al. 2004. Complex haplotypes of the PGC-1alpha gene are associated with carbohydrate metabolism and type 2 diabetes. Diabetes. 53:1385-1393.

9. Bianchi, C., Genova, M.L., Parenti Castelli, G., and Lenaz, G. 2004. The mitochondrial respiratory chain is partially organized in a supercomplex assembly: kinetic evidence using flux control analysis. J. Biol. Chem. 279:36562-36569.

10. de Bakker, P.I., et al. 2005. Efficiency and power in genetic association studies. Nat. Genet. 37:1217-1223.

11. Luo, T.H., et al. 2001. A genome-wide search for type II diabetes susceptibility genes in Chinese 
Hans. Diabetologia. 44:501-506.

12. Lindgren, C.M., et al. 2002. Genomewide search for type 2 diabetes mellitus susceptibility loci in Finnish families: the Botnia study. Am. J. Hum. Genet. 70:509-516

13. Bruce, C.R., et al. 2003. Muscle oxidative capacity is a better predictor of insulin sensitivity than lipid status. J. Clin. Endocrinol. Metab. 88:5444-5451.

14. Trounce, I., Byrne, E., and Marzuki, S. 1989. Decline in skeletal muscle mitochondrial respiratory chain function: possible factor in ageing. Lancet. 1:637-639.

15. Petersen, K.F., et al. 2003. Mitochondrial dysfunction in the elderly: possible role in insulin resistance. Science. 300:1140-1142.

16. Huang, X., et al. 1999. Insulin-regulated mitochondrial gene expression is associated with glucose flux in human skeletal muscle. Diabetes. 48:1508-1514.

17. Horenstein, R.B., and Shuldiner, A.R. 2004. Genetics of diabetes. Rev. Endocr. Metab. Disord. 5:25-36.

18. Altshuler, D., et al. 2000. The common PPAR gamma Pro12Ala polymorphism is associated with decreased risk of type 2 diabetes. Nat. Genet. 26:76-80.

19. Horikawa, Y., et al. 2000. Genetic variation in the gene encoding calpain-10 is associated with type 2 diabetes mellitus. Nat. Genet. 26:163-175.

20. Poulsen, P., et al. 2005. Heritability of insulin secretion, peripheral and hepatic insulin action, and intracellular glucose partitioning in young and old Danish twins. Diabetes. 54:275-283.

21. Petersen, K.F., Dufour, S., and Shulman, G.I. 2005. Decreased insulin-stimulated ATP synthesis and phosphate transport in muscle of insulin-resistant offspring of type 2 diabetic parents. PLoS Med. 2:e233.

22. Morino, K., et al. 2005. Reduced mitochondrial density and increased IRS-1 serine phosphorylation in muscle of insulin-resistant offspring of type 2 diabetic parents. J. Clin. Invest. 115:3587-3593. doi:10.1172/JCI25151.

23. Saxena, R., et al. 2007. Genome-wide association analysis identifies loci for type 2 diabetes and triglyceride levels. Science. 316:1331-1336.

24. Sladek, R., et al. 2007. A genome-wide association study identifies novel risk loci for type 2 diabetes. Nature. 445:881-885.

25. Zeggini, E., et al. 2007. Replication of genome-wide association signals in UK samples reveals risk loci for type 2 diabetes. Science. 316:1336-1341.

26. Scott, L.J., et al. 2007. A genome-wide association study of type 2 diabetes in Finns detects multiple susceptibility variants. Science. 316:1341-1345.

27. Steinthorsdottir, V., et al. 2007. A variant in CDKAL1 influences insulin response and risk of type 2 diabetes. Nat. Genet. 39:770-775.

28. Boushel, R., et al. 2007. Patients with type 2 diabetes have normal mitochondrial function in skeletal muscle. Diabetologia. 50:790-796.

29. Kelley, D.E., He, J., Menshikova, E.V., and Ritov, V.B. 2002. Dysfunction of mitochondria in human skeletal muscle in type 2 diabetes. Diabetes. 51:2944-2950.

30. Ritov, V.B., et al. 2005. Deficiency of subsarcolemmal mitochondria in obesity and type 2 diabetes. Diabetes. 54:8-14.

31. Bua, E., et al. 2006. Mitochondrial DNA-deletion mutations accumulate intracellularly to detrimental levels in aged human skeletal muscle fibers. Am. J. Hum. Genet. 79:469-480.

32. Kujoth, G.C., et al. 2005. Mitochondrial DNA mutations, oxidative stress, and apoptosis in mammalian aging. Science. 309:481-484.

33. Valerio, A., et al. 2006. TNF-alpha downregulates eNOS expression and mitochondrial biogenesis in fat and muscle of obese rodents. J. Clin. Invest. 116:2791-2798. doi:10.1172/JCI28570.

34. Poulsen, P., Levin, K., Beck-Nielsen, H., and Vaag, A. 2002. Age-dependent impact of zygosity and birth weight on insulin secretion and insulin action in twins. Diabetologia. 45:1649-1657.

35. Groop, L., et al. 1996. Metabolic consequences of a family history of NIDDM (the Botnia study): evidence for sex-specific parental effects. Diabetes. 45:1585-1593.

36. Tripathy, D., et al. 2000. Insulin secretion and insulin sensitivity in relation to glucose tolerance: lessons from the Botnia Study. Diabetes. 49:975-980.

37. Lindholm, E., Agardh, E., Tuomi, T., Groop, L., and Agardh, C.D. 2001. Classifying diabetes according to the new WHO clinical stages. Eur. J. Epidemiol. 17:983-989.

38. Leosdottir, M., Nilsson, P., Nilsson, J.A., Mansson, H., and Berglund, G. 2004. The association between total energy intake and early mortality: data from the Malmo Diet and Cancer Study. J. Intern. Med. 256:499-509.

39. Alberti, K.G., and Zimmet, P.Z. 1998. Definition, diagnosis and classification of diabetes mellitus and its complications. Part 1: diagnosis and classification of diabetes mellitus provisional report of a WHO consultation. Diabet. Med. 15:539-553.

40. Vaag, A., Alford, F., Henriksen, F.L., Christopher, M., and Beck-Nielsen, H. 1995. Multiple defects of both hepatic and peripheral intracellular glucose processing contribute to the hyperglycaemia of NIDDM. Diabetologia. 38:326-336.

41. Hemmila, I., Dakubu, S., Mukkala, V.M., Siitari, H., and Lovgren, T. 1984. Europium as a label in time-resolved immunofluorometric assays. Anal. Biochem. 137:335-343.

42. Hother-Nielsen, O., and Beck-Nielsen, H. 1990. On the determination of basal glucose production rate in patients with type 2 (non-insulin-dependent) diabetes mellitus using primed-continuous $3-3 \mathrm{H}$ glucose infusion. Diabetologia. 33:603-610.

43. Markuns, J.F., Wojtaszewski, J.F., and Goodyear, L.J. 1999. Insulin and exercise decrease glycogen synthase kinase- 3 activity by different mechanisms in rat skeletal muscle. J. Biol. Chem. 274:24896-24900.

44. Vandenplas, S., et al. 1984. Blot hybridisation analysis of genomic DNA. J. Med. Genet. 21:164-172.

45. Li, L.C., and Dahiya, R. 2002. MethPrimer: designing primers for methylation PCRs. Bioinformatics. 18:1427-1431.

46. Liang, K.Y., and Zeger, S.L. 1993. Regression analysis for correlated data. Annu. Rev. Public Health. 14:43-68.

47. Zeger, S.L., and Liang, K.Y. 1986. Longitudinal data analysis for discrete and continuous outcomes. Biometrics. 42:121-130.

48. Storgaard, H., Poulsen, P., Ling, C., Groop, L., and Vaag, A.A. 2006. Genetic and nongenetic determinants of skeletal muscle glucose transporter 4 messenger ribonucleic acid levels and insulin action in twins. J. Clin. Endocrinol. Metab. 91:702-708.

49. Purcell, S., Cherny, S.S., and Sham, P.C. 2003. Genetic Power Calculator: design of linkage and association genetic mapping studies of complex traits. Bioinformatics. 19:149-150.

50. Barrett, J.C., Fry, B., Maller, J., and Daly, M.J. 2005. Haploview: analysis and visualization of LD and haplotype maps. Bioinformatics. 21:263-265.

51. Gabriel, S.B., et al. 2002. The structure of haplotype blocks in the human genome. Science. 296:2225-2229. 